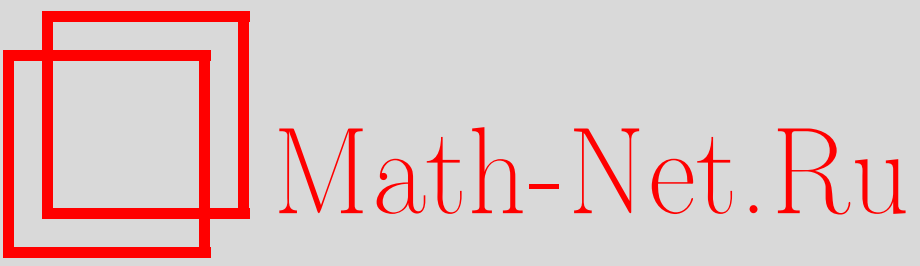

В. Е. Федоров, А. В. Нагуманова, Линейные обратные задачи для одного класса вырожденных эволюционных уравнений дробного порядка, Итоги науки и техн. Сер. Соврем. мат. и ее прил. Темат. обз., 2019, том 167, 97-111

DOI: https://doi.org/10.36535/0233-6723-2019-167-97-111

Использование Общероссийского математического портала Math-Net.Ru подразумевает, что вы прочитали и согласны с пользовательским соглашением

http: //www.mathnet.ru/rus/agreement

Параметры загрузки:

IP : 54.81 .137 .203

26 апреля 2023 г., 04:59:40 


\title{
ЛИНЕЙНЫЕ ОБРАТНЫЕ ЗАДАЧИ \\ ДЛЯ ОДНОГО КЛАССА ВЫРОЖДЕННЫХ ЭВОЛЮЦИОННЫХ УРАВНЕНИЙ ДРОБНОГО ПОРЯДКА
}

\author{
(c) 2019 г. $\quad$ В. Е. ФЕДОРОВ, А. В. НАГУМАНОВА
}

\begin{abstract}
АннотАция. В работе исследуется однозначная разрешимость линейных обратных коэффициентных задач с не зависящим от времени неизвестным коэффициентом для эволюционного уравнения в банаховом пространстве с вырожденным оператором при дробной производной Герасимова-Капуто. Полученные абстрактные результаты использованы при исследовании обратных задач с неопределенным коэффициентом, зависящим только от пространственных переменных, для уравнений с многочленами от самосопряженного эллиптического дифференциального оператора по пространственным переменным. Общие результаты применены также к исследованию однозначной разрешимости обратной задачи для системы уравнений Соболева дробного порядка по времени.
\end{abstract}

Ключевые слова: обратная коэффициентная задача, вырожденное эволюционное уравнение, дробная производная Герасимова-Капуто.

\section{INVERSE LINEAR PROBLEMS FOR A CERTAIN CLASS OF DEGENERATE FRACTIONAL EVOLUTION EQUATIONS}

\author{
(c) 2019 V. E. FEDOROV, A. V. NAGUMANOVA
}

\begin{abstract}
In this paper, we study the unique solvability of linear inverse coefficient problems with a time-independent unknown coefficient for evolution equations in Banach spaces with degenerate operators acting on the Gerasimov-Caputo fractional derivative. We apply abstract results obtained in the paper to the study of inverse problems with undetermined coefficients depending only on spatial variables for equations with polynomials on a self-adjoint, elliptic differential operator with respect to spatial variables. Also, we apply general results to the study of the unique solvability of inverse problems for time-fractional Sobolev systems.
\end{abstract}

Keywords and phrases: inverse coefficient problem, degenerate evolution equation, GerasimovCaputo fractional derivative.

AMS Subject Classification: 35R30, 35R11, 34K29

1. Введение. Пусть $\mathcal{U}, \mathcal{X}, \mathcal{Y}$ - банаховы пространства. Через $\mathcal{L}(\mathcal{X} ; \mathcal{Y})$ будем обозначать банахово пространство линейных непрерывных операторов, действующих из $\mathcal{X}$ в $\mathcal{Y}$. Множество линейных замкнутых операторов с областями определения, плотными в пространстве $\mathcal{X}$, действующих в $\mathcal{Y}$, обозначим через $\mathcal{C l}(\mathcal{X} ; \mathcal{Y})$. При $\mathcal{X}=\mathcal{Y}$ эти обозначения примут вид $\mathcal{L}(\mathcal{X})$ и $\mathcal{C l}(\mathcal{X})$ соответственно.

Работа выполнена при поддержке Правительства РФ (Постановление № 211 от 16.03.2013, соглашение № 02.А03.21.0011) и Министерства образования и науки РФ (задание № 1.6462.2017/БЧ).. 
Пусть $L \in \mathcal{L}(\mathcal{X} ; \mathcal{Y})$, $\operatorname{ker} L \neq\{0\}$, оператор $M \in \mathcal{C l}(\mathcal{X} ; \mathcal{Y})$ имеет область определения $D_{M}$. Рассмотрим вырожденное эволюционное уравнение дробного порядка

$$
D_{t}^{\alpha} L x(t)=M x(t)+B(t) u+y(t), \quad t \in[0, T] ;
$$

здесь $D_{t}^{\alpha}$ - дробная производная Герасимова-Капуто, $B:[0, T] \rightarrow \mathcal{L}(\mathcal{U} ; \mathcal{Y}), y:[0, T] \rightarrow \mathcal{Y}, u \in \mathcal{U}$, $T>0$. Для уравнения (1.1) зададим условия Коши

$$
x^{(k)}(0)=x_{k}, \quad k=0,1, \ldots, m-1,
$$

и условие переопределения

$$
\int_{0}^{T} x(t) d \mu(t)=x_{T} .
$$

Предполагается, что скалярная функция $\mu(t)$ имеет ограниченную вариацию на $[0, T]$. Интеграл в условии (1.3) понимается как векторный интеграл Римана-Стильтьеса. Элементы $x_{k}, k=$ $0,1, \ldots, m-1, x_{T}$ считаются известными.

Задача нахождения функции $x(t)$ из соотношений $(1.1),(1.2)$ называется прямой задачей (или задачей Коши), а задача нахождения пары $(x(t), u)$ из соотношений $(1.1)-(1.3)$ - обратной задачей. Ее решением часто будем называть элемент $u$.

Главная цель данной работы - исследование обратной задачи (1.1)-(1.3) и некоторых близких к ней обратных задач для уравнения (1.1).

Обратные задачи для систем, которые описываются дифференциальными уравнениями, являются важным инструментом для исследований в области геофизики, астрономии, спектрального анализа, при решении различных прикладных проблем (см. [29, 38]). Рассмотрение дифференциальных уравнений в банаховых пространствах позволяет эффективно изучать широкие классы задач для дифференциальных уравнений и систем дифференциальных уравнений в частных производных. Обратные задачи для дифференциальных уравнений первого порядка в банаховых пространствах, разрешенных относительно производной, и для их конкретных реализаций исследовались в $[11,12,15,16,34,38]$ и многих других работах. Обратные задачи для дробных дифференциальных уравнений в банаховых пространствах изучались в $[3,4,35,36]$ и др.

Многие реальные процессы описываются дифференциальными уравнениями с вырожденным оператором при производной высшего порядка по времени (см. $[6,13,30,39])$. Обратные задачи для линейных уравнений первого порядка такого типа изучались в работах Н. Л. Абашеевой [26], В. Е. Федорова, А. В. Уразаевой и Н. Д. Ивановой $[7,10,19,20,24,25,31,33]$, А. Фавини и М. Аль Хорани [27], М. В. Фалалеева [21]. В [32] исследован класс обратных задач для уравнений вида (1.1) с неизвестным параметром $u=u(t)$, зависящим от времени. Такие задачи имеют свою специфику, в частности, данные переопределения также зависят от времени $\left(x_{T}=x_{T}(t)\right)$, и условие переопределения имеет соответствующую структуру. Получены абстрактные результаты об однозначной разрешимости обратных задач, которые были применены к исследованию начальнокраевой задачи для уравнения Соболева дробного порядка с неизвестным коэффициентом, зависящим от времени, при заданной вектор-функции, зависящей от времени и пространственных переменных, в правой части.

Цель настоящей работы - продолжить изучение обратных задач для уравнений в банаховых пространствах с вырожденным оператором в дробной производной. В работе исследуется однозначная разрешимость линейных обратных коэффициентных задач вида (1.2), (1.3) с не зависящим от времени неизвестным коэффициентом $u$ для эволюционного уравнения (1.1) в банаховом пространстве с вырожденным оператором $L$ при дробной производной Герасимова-Капуто. Оператор $M$ предполагается при этом $(L, p)$-ограниченным (см. [39]). В качестве начальных условий используются как условия Коши, так и обобщенные условия Шоуолтера-Сидорова, представляющие собой начальные условия не для всей неизвестной функции, а для ее проекции на фазовое пространство уравнения $D_{t}^{\alpha} L x(t)=M x(t)$ (см. [22]). При этом рассмотрены задачи с условием переопределения для всей искомой функции, для ее проекции на фазовое пространство, а также для ее проекции на подпространство вырождения уравнения - дополнение к фазовому пространству. 
Полученные абстрактные результаты использованы при исследовании обратных задач с неопределенным коэффициентом, зависящим только от пространственных переменных, для уравнений с многочленами от самосопряженного эллиптического дифференциального оператора по пространственным переменным. Частными случаями таких уравнений являются уравнение БаренблаттаЖелтова-Кочиной, уравнение Буссинеска и другие уравнения математической физики. Кроме того, общие результаты использованы для исследования однозначной разрешимости обратной задачи для системы уравнений Соболева дробного порядка по времени с зависящим только от пространственных переменных неопределенным коэффициентом, на который действует дифференциальный оператор.

2. Обратная задача для невырожденного дробного уравнения. Введем обозначения $g_{\delta}(t):=t^{\delta-1} / \Gamma(\delta)$ при $\delta>0, t>0$,

$$
J_{t}^{\delta} h(t):=\left(g_{\delta} * h\right)(t):=\int_{0}^{t} g_{\delta}(t-s) h(s) d s .
$$

Пусть $\alpha>0, m$ - наименьшее целое число, не превосходимое числом $\alpha, D_{t}^{m}$ - обычная производная порядка $m \in \mathbb{N}, J_{t}^{0}$ - тождественный оператор, $D_{t}^{\alpha}$ - дробная производная ГерасимоваКапуто (см. $[2,28])$, т.е.

$$
D_{t}^{\alpha} h(t)=D_{t}^{m} J_{t}^{m-\alpha}\left(h(t)-\sum_{k=0}^{m-1} h^{(k)}(0) g_{k+1}(t)\right) .
$$

Рассмотрим сначала уравнение, разрешенное относительно дробной производной:

$$
D_{t}^{\alpha} z(t)=A z(t)+B(t) u+f(t), \quad t \in[0, T] ;
$$

зЗдесь $D_{t}^{\alpha}$ - дробная производная Герасимова-Капуто, $A$ - ограниченный оператор в банаховом пространстве $\mathcal{Z}(A \in \mathcal{L}(\mathcal{Z})), T>0$ - число, элемент $u$ принадлежит банахову пространству $\mathcal{U}$, $B \in C([0, T] ; \mathcal{L}(\mathcal{U} ; \mathcal{Z})), f \in C([0, T] ; \mathcal{Z})$. Для уравнения $(2.1)$ зададим условия Коши

$$
z^{(k)}(0)=z_{k}, \quad k=0,1, \ldots, m-1,
$$

и условие переопределения

$$
\int_{0}^{T} z(t) d \mu(t)=z_{T}
$$

Скалярная функция $\mu(t)$ имеет ограниченную вариацию на $[0, T]$, заданы элементы $z_{k} \in \mathcal{Z}$, $k=0,1, \ldots, m-1, z_{T} \in \mathcal{Z}$.

Рассмотрим сначала прямую задачу $(2.1),(2.2)$; элемент $u \in \mathcal{U}$ при этом будем считать известным. Под решением задачи $(2.1),(2.2)$ будем понимать вектор-функцию $z \in C^{m-1}([0, T] ; \mathcal{Z})$, для которой

$$
g_{m-\alpha} *\left(z-\sum_{k=0}^{m-1} z^{(k)}(0) g_{k+1}\right) \in C^{m}([0, T] ; \mathcal{Z})
$$

и выполняются равенства (2.1) и (2.2).

При $\alpha, \beta>0$ введем обозначение для функции Миттаг-Леффлера

$$
E_{\alpha, \beta}(v)=\sum_{n=0}^{\infty} \frac{v^{n}}{\Gamma(\alpha n+\beta)} .
$$

Теорема 2.1 (см. [23,37]). Пусть $A \in \mathcal{L}(\mathcal{Z}), B \in C([0, T] ; \mathcal{L}(\mathcal{U} ; \mathcal{Z})), f \in C([0, T] ; \mathcal{Z}), u \in \mathcal{U}$, $z_{k} \in \mathcal{Z}, k=0,1, \ldots, m-1, z_{T} \in \mathcal{Z}$. Тогда существует единственное решение задачи (2.1), (2.2), причем оно имеет вид

$$
z(t)=\sum_{k=0}^{m-1} t^{k} E_{\alpha, k+1}\left(A t^{\alpha}\right) z_{k}+\int_{0}^{t}(t-s)^{\alpha-1} E_{\alpha, \alpha}\left(A(t-s)^{\alpha}\right)(B(s) u+f(s)) d s .
$$


Теперь будем рассматривать обратную задачу (2.1)-(2.3), считая элемент $u$ в уравнении (2.1) неизвестным. Назовем элемент $u \in \mathcal{U}$ решением задачи (2.1)-(2.3), если соответствующее решение задачи Коши (2.1), (2.2) удовлетворяет условию (2.3).

В силу формулы (2.4) элемент $u$ является решением задачи (2.1)-(2.3) тогда и только тогда, когда он удовлетворяет уравнению

где $\chi(A)$ и $\psi(A)$ определены формулами

$$
\chi(A) u=\psi(A),
$$

$$
\begin{gathered}
\chi(A):=\int_{0}^{T} d \mu(t) \int_{0}^{t}(t-s)^{\alpha-1} E_{\alpha, \alpha}\left(A(t-s)^{\alpha}\right) B(s) d s \in \mathcal{L}(\mathcal{U} ; \mathcal{Z}), \\
\psi(A):=z_{T}-\int_{0}^{T} \sum_{k=0}^{m-1} t^{k} E_{\alpha, k+1}\left(A t^{\alpha}\right) z_{k} d \mu(t)-\int_{0}^{T} d \mu(t) \int_{0}^{t}(t-s)^{\alpha-1} E_{\alpha, \alpha}\left(A(t-s)^{\alpha}\right) f(s) d s \in \mathcal{Z} .
\end{gathered}
$$

Теорема 2.2. Пусть $A \in \mathcal{L}(\mathcal{Z}), B \in C([0, T] ; \mathcal{L}(\mathcal{U} ; \mathcal{Z})), f \in C([0, T] ; \mathcal{Z})$, функиия $\mu:[0, T] \rightarrow \mathbb{R}$ имеет ограниченную вариачию. Тогда для однозначной разрешимости задачи (2.1)-(2.3) необходимо и достаточно, чтобы существовал непрерывный обратный оператор $\chi(A)^{-1} \in \mathcal{L}(\mathcal{Z} ; \mathcal{U})$. При этом решение имеет вид $u=\chi(A)^{-1} \psi(A)$ и удовлетворяет неравенству

$$
\|u\|_{\mathcal{U}} \leqslant C\left(\sum_{k=0}^{m-1}\left\|z_{k}\right\|_{\mathcal{Z}}+\left\|z_{T}\right\|_{\mathcal{Z}}+\|f\|_{C([0, T] ; \mathcal{Z})}\right) .
$$

Доказательство. По теореме 2.1 решение задачи Коши $(2.1),(2.2)$ существует при любых $z_{k} \in \mathcal{Z}$, $k=0,1, \ldots, m-1, u \in \mathcal{U}$, и при этом имеет вид (2.4). Подставим решение (2.4) в условие (2.3):

$$
\int_{0}^{T} d \mu(t)\left(\sum_{k=0}^{m-1} t^{k} E_{\alpha, k+1}\left(A t^{\alpha}\right) z_{k}+\int_{0}^{t}(t-s)^{\alpha-1} E_{\alpha, \alpha}\left(A(t-s)^{\alpha}\right)(B(s) u+f(s)) d s\right)=z_{T} .
$$

Отсюда получим формулу (2.5), из которой следует требуемое. Отметим, что если $\chi(A)^{-1} \in$ $\mathcal{L}(\mathcal{Z} ; \mathcal{U})$, то в силу теоремы Банаха об обратном операторе для $u$ справедлива оценка устойчивости $(2.6)$.

\section{3. Обратные задачи для вырожденного уравнения с дробной производной.}

3.1. Задачи с условиями согласования. Пусть $\mathcal{X}, \mathcal{Y}$-банаховы пространства, оператор $L$ принадлежит пространству $\mathcal{L}(\mathcal{X} ; \mathcal{Y}), \operatorname{ker} L \neq\{0\}$, оператор $M \in \mathcal{C l}(\mathcal{X} ; \mathcal{Y})$ имеет область определения $D_{M}$. Введем обозначения

$\rho^{L}(M):=\left\{\mu \in \mathbb{C}:(\mu L-M)^{-1} \in \mathcal{L}(\mathcal{Y} ; \mathcal{X})\right\}, \quad R_{\mu}^{L}(M):=(\mu L-M)^{-1} L, \quad L_{\mu}^{L}(M):=L(\mu L-M)^{-1}$.

Оператор $M$ называется $(L, \sigma)$-ограниченным, если существует такое $a>0$, что для любого $\mu \in \mathbb{C}$ из $|\mu|>a$ вытекает $\mu \in \rho^{L}(M)$. Для $(L, \sigma)$-ограниченного оператора $M$ выберем в комплексной плоскости $\mathbb{C}$ замкнутый контур $\gamma=\{\mu \in \mathbb{C}:|\mu|=r>a\}$. Тогда имеют смысл следующие интегралы как интегралы от аналитических функций по замкнутому контуру:

$$
P:=\frac{1}{2 \pi i} \int_{\gamma} R_{\mu}^{L}(M) d \mu \in \mathcal{L}(\mathcal{X}), \quad Q:=\frac{1}{2 \pi i} \int_{\gamma} L_{\mu}^{L}(M) d \mu \in \mathcal{L}(\mathcal{Y}) .
$$

Положим

$$
\mathcal{X}^{0}:=\operatorname{ker} P, \quad \mathcal{X}^{1}:=\operatorname{im} P, \quad \mathcal{Y}^{0}:=\operatorname{ker} Q, \quad \mathcal{Y}^{1}:=\operatorname{im} Q, \quad P_{0}:=I-P, \quad Q_{0}:=I-Q .
$$

Через $L_{k}$ и $M_{k}$ обозначим сужения операторов $L$ и $M$ на $\mathcal{X}^{k}$ (отметим, что $D_{M_{k}}:=D_{M} \cap \mathcal{X}^{k}$, $k=0,1)$.

Теорема 3.1 (см. $[23,37,39])$. Пусть оператор $M$ является $(L, \sigma)$-ограниченным. Справедливы следующие утверждения: 
(i) $\mathcal{X}=\mathcal{X}^{0} \oplus \mathcal{X}^{1}, \mathcal{Y}=\mathcal{Y}^{0} \oplus \mathcal{Y}^{1}$

(ii) $L_{k} \in \mathcal{L}\left(\mathcal{X}^{k} ; \mathcal{Y}^{k}\right), k=0,1, M_{0} \in \mathcal{C} l\left(\mathcal{X}^{0} ; \mathcal{Y}^{0}\right), M_{1} \in \mathcal{L}\left(\mathcal{X}^{1} ; \mathcal{Y}^{1}\right)$;

(iii) существуют операторы $L_{1}^{-1} \in \mathcal{L}\left(\mathcal{Y}^{1} ; \mathcal{X}^{1}\right)$ u $M_{0}^{-1} \in \mathcal{L}\left(\mathcal{Y}^{0} ; \mathcal{X}^{0}\right)$;

(iv) при $\alpha, \beta>0$ семейства операторов

$$
X_{\alpha, \beta}(t)=\frac{1}{2 \pi i} \int_{\gamma} R_{\lambda}^{L}(M) E_{\alpha, \beta}\left(\lambda t^{\alpha}\right) d \lambda \in \mathcal{L}(\mathcal{X}), \quad Y_{\alpha, \beta}(t)=\frac{1}{2 \pi i} \int_{\gamma} L_{\lambda}^{L}(M) E_{\alpha, \beta}\left(\lambda t^{\alpha}\right) d \lambda \in \mathcal{L}(\mathcal{Y})
$$

являются аналитическими на множестве $\mathbb{C} \backslash\{t \in \mathbb{C}: \operatorname{Re} t \leqslant 0\}$. Кроме того,

$$
X_{\alpha, \beta}(t)=E_{\alpha, \beta}\left(L_{1}^{-1} M_{1} t^{\alpha}\right) P .
$$

Введем обозначение $H:=M_{0}^{-1} L_{0}$. При $p \in \mathbb{N}_{0}:=\{0\} \cup \mathbb{N}$ оператор $M$ называется $(L, p)$ ограниченным, если он $(L, \sigma)$-ограничен, $H^{p} \neq \mathbb{O}$ и $H^{p+1}=\mathbb{O}$.

Рассмотрим вырожденное дробное дифференциальное уравнение

$$
D_{t}^{\alpha} L x(t)=M x(t)+B(t) u+y(t), \quad t \in[0, T],
$$

где $B \in C([0, T] ; \mathcal{L}(\mathcal{U} ; \mathcal{Y})), u \in \mathcal{U}, y \in C([0, T] ; \mathcal{Y})$. Для уравнения (3.1) зададим условия Коши

$$
x^{(k)}(0)=x_{k}, \quad k=0,1, \ldots, m-1,
$$

и условие переопределения

$$
\int_{0}^{T} x(t) d \mu(t)=x_{T}
$$

Сначала предположим, что элемент $u \in \mathcal{U}$ известен, и сформулируем теорему о разрешимости задачи Коши (3.1), (3.2). Решением задачи Коши (3.1), (3.2) называется вектор-функция $x \in C\left([0, T] ; D_{M}\right) \cap C^{m-1}([0, T] ; \mathcal{X})$, для которой

$$
g_{m-\alpha} *\left(L x-\sum_{k=0}^{m-1}(L x)^{(k)}(0) g_{k+1}\right) \in C^{m}([0, T] ; \mathcal{Y})
$$

и выполняются равенства (3.1), (3.2).

Теорема 3.2 (см. $[23,37])$. Пусть оператор $M$ является $(L, p)$-ограниченным, $y \in C([0, T] ; \mathcal{Y})$, $B \in C([0, T] ; \mathcal{L}(\mathcal{U} ; \mathcal{Y}))$, существуют

$$
\left(D_{t}^{\alpha} H\right)^{n} M_{0}^{-1} Q_{0} B \in C([0, T] ; \mathcal{L}(\mathcal{U} ; \mathcal{X})), \quad\left(D_{t}^{\alpha} H\right)^{n} M_{0}^{-1} Q_{0} y \in C([0, T] ; \mathcal{X})
$$

при $n=0,1, \ldots, p, u \in \mathcal{U}, x_{k} \in \mathcal{X}, k=0,1, \ldots, m-1$, и выполняются условия согласования

$$
\left.D_{t}^{k}\right|_{t=0} \sum_{n=0}^{p}\left(D_{t}^{\alpha} H\right)^{n} M_{0}^{-1} Q_{0}(B(t) u+y(t))=-P_{0} x_{k}, \quad k=0,1, \ldots, m-1 .
$$

Тогда существует единственное решение задачи (3.1), (3.2); оно имеет вид

$$
\begin{aligned}
x(t)=\sum_{k=0}^{m-1} t^{k} X_{\alpha, k+1}(t) x_{k}+\int_{0}^{t}(t-s)^{\alpha-1} X_{\alpha, \alpha}(t-s) L_{1}^{-1} Q & (B(s) u+y(s)) d s- \\
& -\sum_{n=0}^{p}\left(D_{t}^{\alpha} H\right)^{n} M_{0}^{-1} Q_{0}(B(t) u+y(s)) .
\end{aligned}
$$


В силу теоремы 3.1 задача (3.1)-(3.3) эквивалентна системе двух задач на взаимно дополнительных подпространствах:

$$
\begin{gathered}
D_{t}^{\alpha} H x^{0}(t)=x^{0}(t)+M_{0}^{-1} Q_{0} B(t) u+M_{0}^{-1} Q_{0} y(t), \\
x^{0(k)}(0)=x_{k}^{0}, \quad k=0,1, \ldots, m-1, \\
\int_{0}^{T} x^{0}(t) d \mu(t)=x_{T}^{0}
\end{gathered}
$$

на подпространстве $\mathcal{X}^{0}$ и

$$
\begin{gathered}
D_{t}^{\alpha} x^{1}(t)=L_{1}^{-1} M_{1} x^{1}(t)+L_{1}^{-1} Q B(t) u+L_{1}^{-1} Q y(t), \\
x^{1(k)}(0)=x_{k}^{1}, \quad k=0,1, \ldots, m-1, \\
\int_{0}^{T} x^{1}(t) d \mu(t)=x_{T}^{1},
\end{gathered}
$$

на подпространстве $\mathcal{X}^{1}$, где $x^{1}(t)=P x(t), x^{0}(t)=P_{0} x(t), x \in[0, T]$.

Введем обозначения

$$
\begin{gathered}
\chi\left(L_{1}^{-1} M_{1}\right):=\int_{0}^{T} d \mu(t) \int_{0}^{t}(t-s)^{\alpha-1} E_{\alpha, \alpha}\left(L_{1}^{-1} M_{1}(t-s)^{\alpha}\right) L_{1}^{-1} Q B(s) d s \in \mathcal{L}\left(\mathcal{U} ; \mathcal{X}^{1}\right), \\
\psi\left(L_{1}^{-1} M_{1}\right):=P x_{T}-\int_{0}^{T} \sum_{k=0}^{m-1} t^{k} E_{\alpha, k+1}\left(L_{1}^{-1} M_{1} t^{\alpha}\right) P x_{k} d \mu(t)- \\
-\int_{0}^{T} d \mu(t) \int_{0}^{t}(t-s)^{\alpha-1} E_{\alpha, \alpha}\left(L_{1}^{-1} M_{1}(t-s)^{\alpha}\right) L_{1}^{-1} Q y(s) d s \in \mathcal{X}^{1} .
\end{gathered}
$$

Теорема 3.3. Пусть оператор $M$ является $(L, p)$-ограниченным, $B \in C([0, T] ; \mathcal{L}(\mathcal{U} ; \mathcal{Y}))$, $y \in C([0, T] ; \mathcal{Y})$, существуют

$$
\left(D_{t}^{\alpha} H\right)^{n} M_{0}^{-1} Q_{0} B \in C([0, T] ; \mathcal{L}(\mathcal{U} ; \mathcal{X})), \quad\left(D_{t}^{\alpha} H\right)^{n} M_{0}^{-1} Q_{0} y \in C([0, T] ; \mathcal{X})
$$

при всех $n=0,1, \ldots, p, \chi\left(L_{1}^{-1} M_{1}\right)^{-1} \in \mathcal{L}\left(\mathcal{X}^{1} ; \mathcal{U}\right)$, функиия $\mu:[0, T] \rightarrow \mathbb{R}$ имеет ограниченную вариачию, $x_{k} \in \mathcal{X}, k=0,1, \ldots, m-1, x_{T} \in \mathcal{X}$ таковы, что выполняются условия согласования

$$
\begin{gathered}
P_{0} x_{k}=-\left.D_{t}^{k}\right|_{t=0} \sum_{n=0}^{p}\left(D_{t}^{\alpha} H\right)^{n} M_{0}^{-1} Q_{0}\left[B(t) \chi\left(L_{1}^{-1} M_{1}\right)^{-1} \psi\left(L_{1}^{-1} M_{1}\right)+y(t)\right], \quad k=0,1, \ldots, m-1, \\
P_{0} x_{T}=-\int_{0}^{T} \sum_{n=0}^{p}\left(D_{t}^{\alpha} H\right)^{n} M_{0}^{-1} Q_{0}\left[B(t) \chi\left(L_{1}^{-1} M_{1}\right)^{-1} \psi\left(L_{1}^{-1} M_{1}\right)+y(t)\right] d \mu(t) .
\end{gathered}
$$

Тогда задача (3.1)-(3.3) имеет единственное решение, при этом

$$
\|u\|_{\mathcal{U}} \leqslant C\left(\sum_{k=0}^{m-1}\left\|P x_{k}\right\|_{\mathcal{X}}+\left\|P x_{T}\right\|_{\mathcal{X}}+\|Q y\|_{C([0, T] ; \mathcal{Y})}\right) .
$$

Доказательство. По теореме 2.2 существует единственное решение задачи (3.8)-(3.10) тогда и только тогда, когда $\chi\left(L_{1}^{-1} M_{1}\right)^{-1} \in \mathcal{L}\left(\mathcal{X}^{1} ; \mathcal{U}\right)$, при этом $u=\chi\left(L_{1}^{-1} M_{1}\right)^{-1} \psi\left(L_{1}^{-1} M_{1}\right)$ и выполняется 
оценка, с учетом непрерывности оператора $L_{1}^{-1}$ эквивалентная (3.13). Согласно [23] существует единственное решение уравнения $(3.5)$ с найденным $u \in \mathcal{U}$, которое имеет вид

$$
x^{0}(t)=-\sum_{n=0}^{p}\left(D_{t}^{\alpha} H\right)^{n} M_{0}^{-1} Q_{0}\left[B(t) \chi\left(L_{1}^{-1} M_{1}\right)^{-1} \psi\left(L_{1}^{-1} M_{1}\right)+y(t)\right] .
$$

Тогда из (3.6), (3.7) следует необходимость выполнения условий (3.11), (3.12) для разрешимости задачи (3.5)-(3.7), а значит, и задачи (3.1)-(3.3).

Условия (3.11), (3.12) перестают быть необходимыми, если потребовать, чтобы $\operatorname{im} B(t) \subset \mathcal{Y}^{1}$, $y(t) \in \mathcal{Y}^{1}$ при $t \in[0, T]$.

Теорема 3.4. Пусть оператор $M$ является $(L, p)$-ограниченным, $B \in C([0, T] ; \mathcal{L}(\mathcal{U} ; \mathcal{Y}))$, $\operatorname{im} B \subset \mathcal{Y}^{1}, y \in C\left([0, T] ; \mathcal{Y}^{1}\right)$, функция $\mu:[0, T] \rightarrow \mathbb{R}$ имеет ограниченную вариачию, $x_{k} \in \mathcal{X}^{1}$, $k=0,1, \ldots, m-1, x_{T} \in \mathcal{X}^{1}$. Задача (3.1)-(3.3) имеет единственное решение в том и только в том случае, когда $\chi\left(L_{1}^{-1} M_{1}\right)^{-1} \in \mathcal{L}\left(\mathcal{X}^{1} ; \mathcal{U}\right) ;$ при этом

$$
\|u\|_{\mathcal{U}} \leqslant C\left(\sum_{k=0}^{m-1}\left\|x_{k}\right\|_{\mathcal{X}}+\left\|x_{T}\right\|_{\mathcal{X}}+\|y\|_{C([0, T] ; \mathcal{Y})}\right) .
$$

Доказательство. В условиях данной теоремы $Q_{0} B=\mathbb{O}, Q_{0} y \equiv 0$, поэтому уравнение (3.5) имеет вид $D_{t}^{\alpha} H x^{0}(t)=x^{0}(t)$. В силу нильпотентности оператора $H$ оно имеет только единственное решение. Отсюда и из условий (3.6), (3.7) следует, что $x_{k}, k=0,1, \ldots, m-1, x_{T}$ принадлежат подпространству $\mathcal{X}^{1}$. В таком случае задача (3.1)-(3.3) эквивалентна задаче (3.8)-(3.10). По теореме 2.2 получим требуемое.

При доказательстве двух предыдущих теорем неопределенный коэффициент $u$ выражался из уравнения на подпространстве $\mathcal{X}^{1}$ с помощью условия переопределения. В некоторых ситуациях удобнее поступить по-другому и выразить $u$ из уравнения на подпространстве $\mathcal{X}^{0}$. Для этого введем обозначение

$$
F_{z}:=\sum_{n=0}^{p} \int_{0}^{T}\left(D_{t}^{\alpha} H\right)^{n} M_{0}^{-1} Q_{0} z(t) d \mu(t) .
$$

Теорема 3.5. Пусть оператор $M$ является $(L, p)$-ограниченным, $B \in C([0, T] ; \mathcal{L}(\mathcal{U} ; \mathcal{Y}))$, $y \in C([0, T] ; \mathcal{Y})$, существуют

$$
\left(D_{t}^{\alpha} H\right)^{n} M_{0}^{-1} Q_{0} B \in C([0, T] ; \mathcal{L}(\mathcal{U} ; \mathcal{X})), \quad\left(D_{t}^{\alpha} H\right)^{n} M_{0}^{-1} Q_{0} y \in C([0, T] ; \mathcal{X})
$$

при всех $n=0,1, \ldots, p, F_{B}^{-1} \in \mathcal{L}\left(\mathcal{X}^{0} ; \mathcal{U}\right)$, функиия $\mu:[0, T] \rightarrow \mathbb{R}$ имеет ограниченную вариацию, $x_{k} \in \mathcal{X}, k=0,1, \ldots, m-1, x_{T} \in \mathcal{X}$ таковы, что выполняются условия согласования

$$
\begin{gathered}
P_{0} x_{k}=\left.D_{t}^{k}\right|_{t=0} \sum_{n=0}^{p}\left(D_{t}^{\alpha} H\right)^{n} M_{0}^{-1} Q_{0}\left[B(t) F_{B}^{-1}\left(P_{0} x_{T}+F_{y}\right)-y(t)\right], \quad k=0,1, \ldots, m-1, \\
-\chi\left(L_{1}^{-1} M_{1}\right) F_{B}^{-1}\left(P_{0} x_{T}+F_{y}\right)=\psi\left(L_{1}^{-1} M_{1}\right) .
\end{gathered}
$$

Тогда задача (3.1)-(3.3) имеет единственное решение, при этом

$$
\|u\|_{\mathcal{U}} \leqslant C\left(\left\|P_{0} x_{T}\right\|_{\mathcal{X}}+\left\|Q_{0} y\right\|_{C([0, T] ; \mathcal{Y})}\right) .
$$

Доказательство. В силу нильпотентности оператора $H$ существует единственное решение уравнения (3.5), которое имеет вид

$$
x^{0}(t)=-\sum_{n=0}^{p}\left(D_{t}^{\alpha} H\right)^{n} M_{0}^{-1} Q_{0}[B(t) u+y(t)] .
$$

Отсюда в силу (3.7) получаем равенство

$$
u=-F_{B}^{-1}\left(P_{0} x_{T}+F_{y}\right)
$$


а в силу (3.6) - необходимость условия согласования (3.14). По теореме 2.2 задача (3.8), (3.9) однозначно разрешима, а условие (3.10) влечет необходимость условия (3.15).

3.2. Задачи без условий согласования. Кроме задачи Коши для уравнения (3.1) рассмотрим также обобщенную задачу Шоуолтера-Сидорова

$$
(P x)^{(k)}=x_{k}, \quad k=0,1, \ldots, m-1,
$$

и сформулируем результат о разрешимости задачи $(3.1),(3.17)$ при известном элементе $u \in \mathcal{U}$.

Решением задачи (3.1), (3.17) называется вектор-функция $x \in C\left([0, T] ; D_{M}\right)$, для которой

$$
L x \in C^{m-1}([0, T] ; \mathcal{Y}), \quad g_{m-\alpha} *\left(L x-\sum_{k=o}^{m-1}(L x)^{(k)}(0) g_{k+1}\right) \in C^{m}([0, T] ; \mathcal{Y}),
$$

и выполняются равенства (3.1) и (3.17). Здесь используется тот факт, что при условии $(L, \sigma)$ ограниченности оператора $M$ гладкость функции $P x$ не меньше гладкости функции $L x$, так как $P x=L_{1}^{-1} Q L x$.

Теорема 3.6 (см. $[23,37])$. Предположим, что оператор $M$ является $(L, p)$-ограниченным, $y \in C([0, T] ; \mathcal{Y}), B \in C([0, T] ; \mathcal{L}(\mathcal{U} ; \mathcal{Y}))$, существуют

$$
\left(D_{t}^{\alpha} H\right)^{n} M_{0}^{-1} Q_{0} B \in C([0, T] ; \mathcal{L}(\mathcal{U} ; \mathcal{X})), \quad\left(D_{t}^{\alpha} H\right)^{n} M_{0}^{-1} Q_{0} y \in C([0, T] ; \mathcal{X})
$$

при $n=0,1, \ldots, p, u \in \mathcal{U}, x_{k} \in \mathcal{X}^{1}, k=0,1, \ldots, m-1$. Тогда существует единственное решение задачи (3.1), (3.17), имеюоеее вид (3.4).

Замечание 3.1. В случае $(L, 0)$-ограниченного оператора $M$ обобщенная задача ШоуолтераСидорова (3.17) эквивалентна задаче Шоуолтера-Сидорова

$$
(L x)^{(k)}=y_{k}, \quad k=0,1, \ldots, m-1 .
$$

Действительно, из равенств (3.17) следуют равенства (3.18) с $y_{k}=L x_{k}=L_{1} x_{k}, k=0,1, \ldots, m-1$. Обратное верно, так как из $(L, 0)$-ограниченности оператора $M$ следует, что $L_{0}=\mathbb{O}($ см. [39]), поэтому $L=L_{1} P$ и из равенства $L x=L_{1} P x=y$ следует, что $P x=L_{1}^{-1} y$. Таким образом, $x_{k}=L_{1}^{-1} y_{k}, k=0,1, \ldots, m-1$. Кроме того, $(L, 0)$-ограниченность оператора $M$ влечет равенство $\operatorname{im} L_{1}=\mathcal{Y}^{1}$ (см. [39]), поэтому в условиях (3.18) выбираются $y_{k} \in \mathcal{Y}^{1}, k=0,1, \ldots, m-1$.

Использование начальных условий (3.17) и условия переопределения

$$
\int_{0}^{T} P x(t) d \mu(t)=x_{T}
$$

позволяет в теореме 3.3 избавиться от условий согласования (3.11) и (3.12) соответственно.

Теорема 3.7. Пусть оператор $M$ является $(L, p)$-ограниченным, $B \in C([0, T] ; \mathcal{L}(\mathcal{U} ; \mathcal{Y}))$, $y \in C([0, T] ; \mathcal{Y})$, существуют

$$
\left(D_{t}^{\alpha} H\right)^{n} M_{0}^{-1} Q_{0} B \in C([0, T] ; \mathcal{L}(\mathcal{U} ; \mathcal{X})), \quad\left(D_{t}^{\alpha} H\right)^{n} M_{0}^{-1} Q_{0} y \in C([0, T] ; \mathcal{X})
$$

при всех $n=0,1, \ldots, p, \chi\left(L_{1}^{-1} M_{1}\right)^{-1} \in \mathcal{L}\left(\mathcal{X}^{1} ; \mathcal{U}\right)$, функция $\mu:[0, T] \rightarrow \mathbb{R}$ имеет ограниченную вариачию, $x_{k} \in \mathcal{X}^{1}, k=0,1, \ldots, m-1, x_{T} \in \mathcal{X}^{1}$. Тогда задача (3.1), (3.17), (3.19) имеет единственное решение, причем выполняется (3.13).

Доказательство. Задача (3.1), (3.17), (3.19) с помощью теоремы 3.1 редуцируется к совокупности уравнения (3.5) и задачи (3.8)-(3.10). В остальном рассуждения повторяют доказательство теоремы 3.3 . 
Замечание 3.2. Как и для начальных условий в замечании 3.1 , нетрудно показать, что в случае $(L, 0)$-ограниченного оператора $M$ условие переопределения (3.19) эквивалентно условию

$$
\int_{0}^{T} L x(t) d \mu(t)=y_{T}
$$

с $y_{T}=L_{1} x_{T}$. Это означает, что в условии (3.20) задается $y_{T} \in \mathcal{Y}^{1}=\operatorname{im} L_{1}$ (см. замечание 3.1).

Начальные условия (3.17) и условие переопределения

$$
\int_{0}^{T} P_{0} x(t) d \mu(t)=x_{T}
$$

дают возможность избавиться от условий согласования (3.14) и (3.15) соответственно в теореме 3.5 и получить следующий результат.

Теорема 3.8. Пусть оператор $M$ является $(L, p)$-ограниченным, $B \in C([0, T] ; \mathcal{L}(\mathcal{U} ; \mathcal{Y}))$, $y \in C([0, T] ; \mathcal{Y})$, существуют

$$
\left(D_{t}^{\alpha} H\right)^{n} M_{0}^{-1} Q_{0} B \in C([0, T] ; \mathcal{L}(\mathcal{U} ; \mathcal{X})), \quad\left(D_{t}^{\alpha} H\right)^{n} M_{0}^{-1} Q_{0} y \in C([0, T] ; \mathcal{X})
$$

при всех $n=0,1, \ldots, p, F_{B}^{-1} \in \mathcal{L}\left(\mathcal{X}^{0} ; \mathcal{U}\right)$, функция $\mu:[0, T] \rightarrow \mathbb{R}$ имеет ограниченную вариачию, $x_{k} \in \mathcal{X}^{1}, k=0,1, \ldots, m-1, x_{T} \in \mathcal{X}^{0}$. Тогда задача (3.1), (3.17), (3.21) имеет единственное решение, при этом выполняется (3.16).

Доказательство. Задача также редуцируется к совокупности обратной задачи (3.5), (3.7) и прямой задачи (3.8), (3.9), после чего воспроизводится доказательство теоремы 3.5. При этом необходимости в условиях согласования (3.14) и (3.15) не возникает, как это следует из вида условий (3.17), (3.21).

\section{4. Приложения к уравнениям с многочленами от самосопряженного эллиптического оператора.}

\section{1. Общие результаты. Пусть}

$$
P_{n}(\lambda)=\sum_{i=0}^{n} a_{i} \lambda^{i}, \quad Q_{n}(\lambda)=\sum_{i=0}^{n} b_{i} \lambda^{i}, \quad R_{n}(\lambda)=\sum_{i=0}^{n} c_{i} \lambda^{i},
$$

$a_{i}, b_{i}, c_{i} \in \mathbb{C}, i=0,1, \ldots, n, a_{n} \neq 0$. Пусть, кроме того, $\Omega \subset \mathbb{R}^{d}$ - ограниченная область с гладкой границей $\partial \Omega$, пучок операторов $A, B_{1}, B_{2}, \ldots, B_{r}$ регулярно эллиптичен (см. [17, с. 454]), где

$$
\begin{gathered}
(A u)(x)=\sum_{|q| \leqslant 2 r} a_{q}(x) D_{x}^{q} u(x), \quad a_{q} \in C^{\infty}(\bar{\Omega}), \\
\left(B_{l} u\right)(x)=\sum_{|q| \leqslant r_{l}} b_{l q}(x) D_{x}^{q} u(x), \quad b_{l q} \in C^{\infty}(\partial \Omega), \quad l=1,2, \ldots, r, \\
D_{x}^{q}=D_{x_{1}}^{q_{1}} D_{x_{2}}^{q_{2}} \ldots D_{x_{d}}^{q_{d}}, \quad D_{x_{i}}^{q_{i}}=\frac{\partial^{q_{i}}}{\partial x_{i}^{q_{i}}}, \quad q=\left(q_{1}, q_{2}, \ldots, q_{d}\right) \in \mathbb{N}_{0}^{d} .
\end{gathered}
$$

Пусть оператор $A_{1} \in \mathcal{C l}\left(L_{2}(\Omega)\right), A_{1} u=A u$, с областью определения $D\left(A_{1}\right)=H_{\left\{B_{l}\right\}}^{2 r}(\Omega)($ см. $[17$, c. 399]) самосопряжен и имеет ограниченный справа спектр. В таком случае спектр $\sigma\left(A_{1}\right)$ оператора $A_{1}$ дискретный, вещественный и сгущается к $-\infty$. Пусть $0 \notin \sigma\left(A_{1}\right),\left\{\varphi_{k}: k \in \mathbb{N}\right\}-$ ортонормированная в $L_{2}(\Omega)$ система собственных функций оператора $A_{1}$, занумерованных по 
невозрастанию соответствующих собственных значений $\left\{\lambda_{k}: k \in \mathbb{N}\right\}$ с учетом их кратности. Рассмотрим обратную задачу

$$
\begin{gathered}
D_{t}^{\alpha} P_{n}(A) v(x, t)=Q_{n}(A) v(x, t)+R_{n}(A) u(x)+y(x, t), \quad(x, t) \in \Omega \times[0, T], \\
B_{l} A^{k} v(x, t)=0, \quad k=0,1, \ldots, n-1, \quad l=1,2, \ldots, r, \quad(x, t) \in \partial \Omega \times[0, T], \quad \\
P_{n}(A) v(x, 0)=v_{0}(x), \quad \frac{\partial P_{n}(A) v}{\partial t}(x, 0)=v_{1}(x), \quad \ldots, \quad \frac{\partial^{m-1} P_{n}(A) v}{\partial t^{m-1}}(x, 0)=v_{m-1}(x), \quad x \in \Omega, \\
P_{n}(A) v(x, \tau)=v_{\tau}(x), \quad x \in \Omega,
\end{gathered}
$$

где $\alpha>0, m$ - наименьшее натуральное число, не превосходимое числом $\alpha, D_{t}^{\alpha}$ - дробная производная Герасимова-Капуто по переменной $t$ и $\tau \in(0, T]$.

Положим

$$
\begin{gathered}
\mathcal{X}=\left\{w \in H^{2 r n}(\Omega): B_{l} A^{k} w(x)=0, k=0,1, \ldots, n-1, l=1,2, \ldots, r, x \in \partial \Omega\right\}=\mathcal{U}, \\
\mathcal{Y}=L_{2}(\Omega), \quad L=P_{n}(A), \quad M=Q_{n}(A), \quad B=R_{n}(A) .
\end{gathered}
$$

Тогда $L, M, B \in \mathcal{L}(\mathcal{X} ; \mathcal{Y})$. Уравнение $(4.1)$ является вырожденным, если $\left\{k \in \mathbb{N}: P_{n}\left(\lambda_{k}\right)=0\right\} \neq \emptyset$.

Теорема 4.1 (см. [22]). Пусть спектр $\sigma\left(A_{1}\right)$ не содержит точки 0 и общих корней многочленов $P_{n}(\lambda)$ и $Q_{n}(\lambda)$. Тогда оператор $M$ является $(L, 0)$-ограниченным,

$$
\begin{gathered}
\sigma^{L}(M)=\left\{\mu \in \mathbb{C}: \mu=Q_{n}\left(\lambda_{k}\right) / P_{n}\left(\lambda_{k}\right), \quad P_{n}\left(\lambda_{k}\right) \neq 0\right\}, \\
P=\sum_{P_{n}\left(\lambda_{k}\right) \neq 0}\left\langle\cdot, \varphi_{k}\right\rangle \varphi_{k}, \quad Q=\sum_{P_{n}\left(\lambda_{k}\right) \neq 0}\left\langle\cdot, \varphi_{k}\right\rangle \varphi_{k}, \\
\mathcal{X}^{0}=\mathcal{Y}^{0}=\operatorname{span}\left\{\varphi_{k}: P_{n}\left(\lambda_{k}\right)=0\right\},
\end{gathered}
$$

$\mathcal{X}^{1}$ является замыканием множества $\operatorname{span}\left\{\varphi_{k}: P_{n}\left(\lambda_{k}\right) \neq 0\right\}$ в норме пространства $\mathcal{X}, \mathcal{Y}^{1}-$ замыкание этого же линеала в норме пространства $L_{2}(\Omega)$.

Теорема 4.2. Пусть спектр $\sigma\left(A_{1}\right)$ не содержит точки 0 и общих корней многочленов $P_{n}(\lambda)$ $u Q_{n}(\lambda), c_{n} \neq 0, R_{n}\left(\lambda_{k}\right) \neq 0$ nри $P_{n}\left(\lambda_{k}\right) \neq 0, y \in C\left([0, T] ; L_{2}(\Omega)\right)$, функиии $v_{k}, k=0,1, \ldots, m-1$, $v_{T}$ принадлежат замыканию линеала $\operatorname{span}\left\{\varphi_{k}: P_{n}\left(\lambda_{k}\right) \neq 0\right\}$ в норме пространства $L_{2}(\Omega)$. Тогда задача (4.1)-(4.4) при достаточно малом $\tau \in(0, T]$ имеет единственное решение.

Доказательство. Заметим, что задача (4.1)-(4.4) редуцируется к задаче (3.1), (3.18), (3.20) с $y_{k} \in \operatorname{im} L_{1}=\mathcal{Y}^{1}, k=0,1, \ldots, m-1, y_{T} \in \mathcal{Y}^{1}$ и с $(L, 0)$-ограниченным оператором $M$ в силу теоремы 4.1. Эта задача в силу замечаний $3.1,3.2$ эквивалентна задаче $(3.1),(3.17),(3.19)$ с

$$
x_{k}=L_{1}^{-1} v_{k}=\sum_{P_{n}\left(\lambda_{k}\right) \neq 0} \frac{\left\langle v_{k}, \varphi_{k}\right\rangle \varphi_{k}}{P_{n}\left(\lambda_{k}\right)}, \quad k=0,1, \ldots, m-1, \quad x_{\tau}=L_{1}^{-1} v_{\tau}=\sum_{P_{n}\left(\lambda_{k}\right) \neq 0} \frac{\left\langle v_{\tau}, \varphi_{k}\right\rangle \varphi_{k}}{P_{n}\left(\lambda_{k}\right)} .
$$

Здесь и далее через $\langle\cdot, \cdot\rangle$ обозначается скалярное произведение в пространстве $L_{2}(\Omega)$.

Проверим выполнение условий теоремы 3.7 .

В этой задаче $\mu$ - функция единичного скачка в точке $\tau, B=R_{n}(A)$ не зависит от времени. В условиях данной теоремы

$$
\begin{gathered}
L_{1}^{-1} Q B=\sum_{P_{n}\left(\lambda_{k}\right) \neq 0} \frac{R_{n}\left(\lambda_{k}\right)\left\langle\cdot, \varphi_{k}\right\rangle \varphi_{k}}{P_{n}\left(\lambda_{k}\right)}, \quad(Q B)^{-1}=\sum_{P_{n}\left(\lambda_{k}\right) \neq 0} \frac{\left\langle\cdot, \varphi_{k}\right\rangle \varphi_{k}}{R_{n}\left(\lambda_{k}\right)}, \\
L_{1}^{-1} M_{1}=\sum_{P_{n}\left(\lambda_{k}\right) \neq 0} \frac{Q_{n}\left(\lambda_{k}\right)\left\langle\cdot, \varphi_{k}\right\rangle \varphi_{k}}{P_{n}\left(\lambda_{k}\right)},
\end{gathered}
$$

поэтому в силу того, что $c_{n} \neq 0$, для $z \in \mathcal{Y}^{1}$ имеем

$$
\left\|(Q B)^{-1} z\right\|_{\mathcal{U}}^{2}=\sum_{P_{n}\left(\lambda_{k}\right) \neq 0} \frac{\left(1+\left|\lambda_{k}\right|^{2 n}\right)\left|\left\langle z, \varphi_{k}\right\rangle\right|^{2}}{\left|R_{n}\left(\lambda_{k}\right)\right|^{2}} \leqslant C \sum_{P_{n}\left(\lambda_{k}\right) \neq 0}\left|\left\langle z, \varphi_{k}\right\rangle\right|^{2}=\|z\|_{\mathcal{Y}^{1}},
$$


Таким образом, $(Q B)^{-1} \in \mathcal{L}\left(\mathcal{Y}^{1} ; \mathcal{U}\right)$. Оператор

$$
\begin{aligned}
\chi\left(L_{1}^{-1} M_{1}\right)=\int_{0}^{\tau} t^{\alpha-1} E_{\alpha, \alpha}\left(t^{\alpha} L_{1}^{-1} M_{1}\right) d t \sum_{P_{n}\left(\lambda_{k}\right) \neq 0} \frac{R_{n}\left(\lambda_{k}\right)\left\langle\cdot, \varphi_{k}\right\rangle \varphi_{k}}{P_{n}\left(\lambda_{k}\right)}= \\
\quad=\frac{\tau^{\alpha}}{\Gamma(\alpha+1)}\left(I+\tau^{\alpha} \Gamma(\alpha+1) L_{1}^{-1} M_{1} E_{\alpha, 2 \alpha+1}\left(\tau^{\alpha} L_{1}^{-1} M_{1}\right)\right) \sum_{P_{n}\left(\lambda_{k}\right) \neq 0} \frac{R_{n}\left(\lambda_{k}\right)\left\langle\cdot, \varphi_{k}\right\rangle \varphi_{k}}{P_{n}\left(\lambda_{k}\right)}
\end{aligned}
$$

с учетом ограниченности оператора $L_{1}^{-1} M_{1}$ непрерывно обратим при достаточно малом $\tau>0$, так как в этом случае непрерывно обратим оператор

$$
I+\tau^{\alpha} \Gamma(\alpha+1) L_{1}^{-1} M_{1} E_{\alpha, 2 \alpha+1}\left(\tau^{\alpha} L_{1}^{-1} M_{1}\right)
$$

являющийся малым возмущением тождественного оператора.

Рассмотрим другое условие переопределения:

$$
\int_{0}^{T} \sum_{P_{n}\left(\lambda_{k}\right)=0}\left\langle v(\cdot, t), \varphi_{k}\right\rangle \varphi_{k}(x) d t=v_{T}(x), \quad x \in \Omega .
$$

Теорема 4.3. Пусть спектр $\sigma\left(A_{1}\right)$ не содержит точки 0 и общих корней многочленов $P_{n}(\lambda)$ и $Q_{n}(\lambda), R_{n}\left(\lambda_{k}\right) \neq 0$ при $P_{n}\left(\lambda_{k}\right)=0, y \in C\left([0, T] ; L_{2}(\Omega)\right)$, функции $v_{k}, k=0,1, \ldots, m-1$, принадлежат замыканию линеала $\operatorname{span}\left\{\varphi_{k}: P_{n}\left(\lambda_{k}\right) \neq 0\right\}$ в норме пространства $L_{2}(\Omega)$, $v_{T} \in \operatorname{span}\left\{\varphi_{k}: P_{n}\left(\lambda_{k}\right)=0\right\}$. Тогда задача (4.1)-(4.3), (4.5) имеет единственное решение.

Доказательство. Рассуждая, как при доказательстве предыдущей теоремы, проверим выполнение условий теоремы 3.8. Имеем $\mu(t)=t$; тот же самый оператор $B=R_{n}(A)$ с обратным

$$
\left(Q_{0} B\right)^{-1}=\sum_{P_{n}\left(\lambda_{k}\right)=0} \frac{\left\langle\cdot, \varphi_{k}\right\rangle \varphi_{k}}{R_{n}\left(\lambda_{k}\right)} \in \mathcal{L}\left(\mathcal{Y}^{0} ; \mathcal{U}\right)
$$

При этом учитывается конечность этой суммы, так как число корней многочлена конечно. Кроме того,

$$
F_{B}=\sum_{P_{n}\left(\lambda_{k}\right)=0} \frac{R_{n}\left(\lambda_{k}\right)\left\langle\cdot, \varphi_{k}\right\rangle \varphi_{k}}{Q_{n}\left(\lambda_{k}\right)} T \in \mathcal{L}\left(\mathcal{U} ; \mathcal{X}^{0}\right), \quad F_{B}^{-1}=\frac{1}{T} \sum_{P_{n}\left(\lambda_{k}\right)=0} \frac{Q_{n}\left(\lambda_{k}\right)\left\langle\cdot, \varphi_{k}\right\rangle \varphi_{k}}{R_{n}\left(\lambda_{k}\right)} \in \mathcal{L}\left(\mathcal{X}^{0} ; \mathcal{U}\right) .
$$

По теореме 3.8 получим требуемое.

\section{2. Примеры. Возьмем}

$$
P_{1}(\lambda)=a-\lambda, \quad Q_{1}(\lambda)=b_{1} \lambda+b_{0}, \quad R_{1}(\lambda)=c_{1} \lambda+c_{0}, \quad A u=\Delta u, \quad r=1, \quad B_{1}=I .
$$

Тогда рассмотренные выше задачи примут вид обратной задачи

$$
\begin{gathered}
D_{t}^{\alpha}(a-\Delta) v(x, t)=\left(b_{1} \Delta+b_{0}\right) v(x, t)+\left(c_{1} \Delta+c_{0}\right) u(x)+y(x, t), \quad(x, t) \in \Omega \times[0, T], \\
v(x, t)=0, \quad(x, t) \in \partial \Omega \times[0, T], \\
\frac{\partial^{k} v}{\partial t^{k}}(x, t)=v_{k}(x), \quad k=0,1, \ldots, m-1, \quad x \in \Omega,
\end{gathered}
$$

с условием переопределения

$$
(a-\Delta) v(x, \tau)=v_{\tau}(x), \quad x \in \Omega,
$$

или

$$
\int_{0}^{T} \sum_{\lambda_{k}=a}\left\langle v(x, t), \varphi_{k}\right\rangle \varphi_{k}(x) d t=v_{T}(x), \quad x \in \Omega
$$

При $\alpha=1, b_{1} \neq 0, b_{0}=0$ уравнение (4.6) является уравнением Баренблатта-ЖелтоваКочиной, описывающим фильтрацию в трещиновато-пористой среде (см. [1]), при $\alpha=1, b_{1}=0$, 
$b_{0} \neq 0$ - это уравнение переходных процессов в полупроводниках (см. [13]), при $\alpha=2$ - это уравнение Буссинеска, возникающее при описании продольных волн в стержнях, в теории длинных волн на воде, при описании волн в плазме (см. $[6,8,18])$.

Теоремы 4.2 и 4.3 позволяют утверждать об однозначной разрешимости задач (4.6)-(4.9) и $(4.6)-(4.8),(4.10)$ в случае любого $\alpha>0$, в том числе дробного.

Условия теоремы 4.2 о существовании единственного решения задачи (4.6)-(4.9) означают, что $b_{1} a+b_{0} \neq 0, c_{1} \neq 0, c_{1} \lambda_{k}+c_{0} \neq 0$ при $\lambda_{k} \neq a$, число $\tau \in(0, T]$ достаточно мало.

Согласно теореме 4.3 для однозначной разрешимости задачи (4.6)-(4.8), (4.10) должны выполняться условия $b_{1} a+b_{0} \neq 0, c_{1} a+c_{0} \neq 0$.

Рассмотрим конкретный пример. Пусть

$$
P_{1}(\lambda)=1+\lambda, \quad Q_{1}(\lambda)=\lambda, \quad R_{1}(\lambda)=1-\lambda, \quad d=1, \quad \Omega=(0, \pi), \quad A u=u^{\prime \prime} .
$$

Тогда $\lambda_{k}=-k^{2}, \varphi_{k}(x)=\sin k x, k \in \mathbb{N}$.

В силу сказанного выше по теореме 4.2 однозначно разрешима при малых $\tau \in(0, T]$ задача (4.6)-(4.9), которая в данном случае имеет вид

$$
\begin{gathered}
D_{t}^{\alpha}\left(1+\frac{\partial^{2}}{\partial x^{2}}\right) v(x, t)=\frac{\partial^{2}}{\partial x^{2}} v(x, t)+\left(1-\frac{\partial^{2}}{\partial x^{2}}\right) u(x)+y(x, t), \quad(x, t) \in(0, \pi) \times[0, T], \\
v(0, t)=v(\pi, t)=0, \quad t \in[0, T], \\
\frac{\partial^{k} v}{\partial x^{k}}(x, t)=v_{k}(x), \quad k=0,1, \ldots, m-1, \quad x \in(0, \pi), \\
\left(1+\frac{\partial^{2}}{\partial x^{2}}\right) v(x, \tau)=v_{\tau}(x), \quad x \in(0, \pi) .
\end{gathered}
$$

При этом $v_{k}, k=0,1, \ldots, m-1, v_{T}$ предполагаются принадлежащими замыканию в норме пространства $L_{2}(0, \pi)$ линеала $\{\sin k x, k=2,3, \ldots\}$.

Если же в этой задаче условие переопределения (4.14) заменить на условие

$$
\int_{0}^{T}\langle v(x, t), \sin x\rangle \sin x d t=v_{T}(x), \quad x \in(0, \pi),
$$

то при $v_{k}, k=0,1, \ldots, m-1$, из замыкания линеала $\{\sin k x, k=2,3, \ldots\}$ в норме $L_{2}(0, \pi), v_{T}(x)=$ $c \sin x$ при некотором $c \in \mathbb{R}$, задача (4.11)-(4.13), (4.15) однозначно разрешима по теореме 4.3.

5. Обратная задача для системы Соболева дробного порядка по времени. Рассмотрим обратную задачу

$$
\begin{gathered}
\frac{\partial^{k} v}{\partial t^{k}}(x, 0)=v_{k}(x), \quad k=0,1, \ldots, m-1, \quad x \in \Omega, \\
v_{n}(x, t):=\sum_{i=1}^{3} v_{i}(x, t) n_{i}(x)=0,(x, t) \in \partial \Omega \times[0, T], \\
v(x, \tau)=v_{\tau}(x), \ldots, x \in \Omega,
\end{gathered}
$$

для системы уравнений Соболева дробного порядка

$$
\begin{gathered}
D_{t}^{\alpha} v(x, t)=[v(x, t), \bar{\omega}]-r(x, t)+\Delta u(x), \quad(x, t) \in \Omega \times[0, T], \\
\nabla \cdot v(x, t)=0, \quad(x, t) \in \Omega \times[0, T],
\end{gathered}
$$

описывающей при $\alpha=1$ динамику малых внутренних движений стратифицированной жидкости в равновесном состоянии (см. [14]). Здесь $m$ - наименьшее натуральное число, не превосходимое числом $\alpha>0, \Omega \subset \mathbb{R}^{3}$ - ограниченная область с границей $\partial \Omega$ класса $C^{\infty}, n=\left(n_{1}, n_{2}, n_{3}\right)$ - вектор внешней нормали к ее границе, $v=\left(v_{1}, v_{2}, v_{3}\right)$ - вектор скорости движения частиц жидкости, $r=$ 
$\left(r_{1}, r_{2}, r_{3}\right)=\left(p_{x_{1}}, p_{x_{2}}, p_{x_{3}}\right)$ - градиент нестационарного давления, $[\cdot, \bar{\omega}]$ - векторное произведение на вектор $\bar{\omega}=(0,0, \omega) \in \mathbb{R}^{3}$, где $\omega$ - удвоенная угловая скорость вращения,

$$
\nabla \cdot v=\frac{\partial v_{1}}{\partial x_{1}}+\frac{\partial v_{2}}{\partial x_{2}}+\frac{\partial v_{3}}{\partial x_{3}}
$$

Неизвестными вектор-функциями являются $v$ и $r$, неопределенный коэффициент-векторфункция $u$.

Введем обозначения $\mathbb{L}_{2}:=\left(L_{2}(\Omega)\right)^{3}, \mathcal{L}:=\left\{v \in\left(C_{0}^{\infty}(\Omega)\right)^{3}: \nabla \cdot v=0\right\}$. Замыкание линеала $\mathcal{L}$ по норме пространства $\mathbb{L}_{2}$ обозначим через $\mathbb{H}_{\sigma}$. Это гильбертово пространство со скалярным произведением $\langle\cdot, \cdot\rangle$ пространства $\mathbb{L}_{2}$. Существует представление $\mathbb{L}_{2}=\mathbb{H}_{\sigma} \oplus \mathbb{H}_{\pi}$, где $\mathbb{H}_{\pi}$ - ортогональное дополнение к $\mathbb{H}_{\sigma}$. Обозначим через $\Pi: \mathbb{L}_{2} \rightarrow \mathbb{H}_{\pi}$ ассоциированный с этим представлением ортопроектор. Далее, $\mathbb{H}^{1}:=\left(H^{1}(\Omega)\right)^{3}, \mathbb{H}_{\sigma}^{1}$ - замыкание линеала $\mathcal{L}$ по норме пространства $\mathbb{H}^{1}$, $\mathbb{H}_{\sigma}^{2}:=\mathbb{H}_{\sigma}^{1} \cap \mathbb{H}^{2}$.

Следуя подходу С. Л. Соболева (см. [14]), используем обобщенную постановку задачи (5.1)(5.5), заменив уравнение несжимаемости (5.5) и граничное условие (5.2) на уравнение

$$
\Pi v(\cdot, t)=0, \quad t \in[0, T] .
$$

Действительно, в силу плотности множества $\left\{\nabla \varphi: \varphi \in C^{\infty}(\Omega)\right\}$ в подпространстве $\mathbb{H}_{\pi}$ и в силу интегрального тождества

$$
\int_{\Omega}\langle v, \nabla \varphi\rangle_{\mathbb{R}^{3}} d x=\int_{\partial \Omega} v_{n} \varphi d s-\int_{\Omega}(\nabla \cdot v) \varphi d x,
$$

справедливого при всех $\varphi \in C^{\infty}(\Omega)$, получим, что для функции $v \in \mathbb{H}^{1}=\left(H^{1}(\Omega)\right)^{3}$ выполнение равенств (5.2) и (5.5) равносильно тому, что $v \in \mathbb{H}_{\sigma}$, т.е. П $v=0$. Отказавшись от ограничения $v \in \mathbb{H}^{1}$, получим условие (5.6).

Очевидно, что оператор $V: v \rightarrow[v, \bar{\omega}], \bar{\omega}=(0,0, \omega)$, осуществляет линейное непрерывное отображение из $\mathbb{L}_{2}$ в $\mathbb{L}_{2}$, при этом $\|V\|_{\mathcal{L}\left(\mathbb{L}_{2}\right)}=|\omega|$.

Положим $\Sigma=I-\Pi, \mathcal{X}=\mathcal{Y}=\mathbb{L}_{2}=\mathbb{H}_{\sigma} \times \mathbb{H}_{\pi}, \mathcal{U}=\mathbb{H}_{\sigma}^{2}, V_{\sigma}=\left.V\right|_{\mathbb{H}_{\sigma}}$,

$$
L=\left(\begin{array}{ll}
I & \mathbb{O} \\
\mathbb{O} & \mathbb{O}
\end{array}\right) \in \mathcal{L}(\mathcal{X}), \quad M=\left(\begin{array}{cc}
\Sigma V_{\sigma} & \mathbb{O} \\
\Pi V_{\sigma} & -I
\end{array}\right) \in \mathcal{L}(\mathcal{X}), \quad B=\Delta \in \mathcal{L}\left(\mathbb{H}_{\sigma}^{2} ; \mathbb{L}_{2}\right) .
$$

Лемма 5.1 (см. [5]). Пусть операторы $L$ и $M$ имеют вид (5.6). Тогда $M-(L, 0)$-ограниченный оператор и

$$
P=\left(\begin{array}{cc}
I & \mathbb{O} \\
\Pi V_{\sigma} & \mathbb{O}
\end{array}\right), \quad Q=\left(\begin{array}{ll}
I & \mathbb{O} \\
\mathbb{O} & \mathbb{O}
\end{array}\right)
$$

В силу леммы 5.1 подпространство $\mathcal{X}^{1}:=\operatorname{im} P$ изоморфно $\mathbb{H}_{\sigma} \times\{0\}$,

$$
\mathcal{X}^{0}:=\operatorname{ker} P=\{0\} \times \mathbb{H}_{\pi}, \quad \mathcal{Y}^{1}:=i m Q=\mathbb{H}_{\sigma} \times\{0\}, \quad \mathcal{Y}^{0}:=\operatorname{ker} Q=\{0\} \times \mathbb{H}_{\pi} .
$$

Теорема 5.1. Для любых $v_{k} \in \mathbb{H}_{\sigma}, k=0,1, \ldots, m-1, v_{\tau} \in \mathbb{H}_{\sigma}$ при достаточно малом $\tau \in$ $(0, T]$ существует единственное решение задачи (5.1), (5.3), (5.4), (5.6).

Доказательство. Из вида проекторов $P, Q$ в лемме 5.1 следует, что задача (5.1), (5.3), (5.4), (5.6) редуцируется к задаче (3.1), (3.17), (3.19). При этом согласно лемме 5.1 оператор $M$ является $(L, 0)$-ограниченным, функция $\mu$ имеет ограниченную вариацию как функция единичного скачка в точке $\tau$, оператор $Q B=\Sigma \Delta: \mathbb{H}_{\sigma}^{2} \rightarrow \mathbb{H}_{\sigma}$ непрерывно обратим (см. [9]), $L_{1}^{-1} M_{1}=\Sigma V_{\sigma}$. Оператор

$$
\begin{aligned}
\chi\left(L_{1}^{-1} M_{1}\right)^{-1}=\tau^{-\alpha} \Gamma(\alpha+1) \Delta^{-1}\left(I+\tau^{\alpha} \Gamma(\alpha+1) L_{1}^{-1} M_{1} E_{\alpha, 2 \alpha+1}\left(\tau^{\alpha} L_{1}^{-1} M_{1}\right)\right)^{-1}= \\
=\tau^{-\alpha} \Gamma(\alpha+1) \Delta^{-1} \sum_{k=0}^{\infty}(-1)^{k} \tau^{\alpha k}\left[\Gamma(\alpha+1) L_{1}^{-1} M_{1} E_{\alpha, 2 \alpha+1}\left(\tau^{\alpha} L_{1}^{-1} M_{1}\right)\right]^{k}
\end{aligned}
$$

существует и непрерывен при достаточно малом $\tau>0$. По теореме 3.7 получим требуемое. 


\section{СПИСОК ЛИТЕРАТУРЫ}

1. Баренблатт Г. И., Желтов Ю. П., Кочина И. Н. Об основных представлениях теории фильтрации однородных жидкостей в трещиноватых породах // Прикл. мат. мех. - 1960. — 24, № 5. - С. $852-864$.

2. Герасимов A. Н. Обобщение линейных законов деформации и их приложение к задачам внутреннего трения// Прикл. мат. мех. - 1948. - 12. - С. 529-539.

3. Глушак A. В. Обратная задача для абстрактного дифференциального уравнения Эйлера-ПуассонаДарбу// Совр. мат. Фундам. напр. - 2006. - 15. - С. 126-141.

4. Глушак A. В. Об одной обратной задаче для абстрактного дифференциального уравнения дробного порядка// Мат. заметки. - 2010. - 87, № 5. - С. 684-693.

5. Гордиевских Д. М., Федоров В. Е. Решения начально-краевых задач для некоторых вырожденных систем уравнений дробного порядка по времени// Изв. Иркут. гос. ун-та. Сер. мат. - 2015. - 12. C. $12-22$.

6. Демиденко Г. В., Успенский С. В. Уравнения и системы, не разрешенные относительно старшей производной. - Новосибирск: Научная книга, 1998.

7. Иванова Н. Д., Федоров B. Е., Комарова K. М. Нелинейная обратная задача для системы Осколкова, линеаризованной в окрестности стационарного решения// Вестн. Челябинск. гос. ун-та. Мат. Мех. Информ. - 2012. - 26 (280), № 15. - С. 49-70.

8. Икези $X$. Экспериментальное исследование солитонов в плазме// в кн.: Солитоны в действии. - М.: Мир, 1981. - С. 163-184.

9. Ладъженская О. А. Математические вопросы динамики вязкой несжимаемой жидкости. - М.: ГИФМЛ, 1961.

10. Плеханова M. B., Федоров B. Е. Оптимальное управление вырожденными распределенными системами. - Челябинск: Издат. центр ЮУрГУ, 2013.

11. Прилепко А. И. Метод полугрупп решения обратных, нелокальных и неклассических задач. Прогнозуправление и прогноз-наблюдение эволюционных уравнений. I// Диффер. уравн. - 2005. — 41, № 11 . - C. $1560-1571$.

12. Пятков С. Г., Самков М. Л. О некоторых классах коэффициентных обратных задач для параболических систем уравнений// Мат. тр. - 2012. - 15, № 1. - С. 155-177.

13. Свешников А. Г., Альшин А. Б., Корпусов М. О., Плетнер Ю. Д. Линейные и нелинейные уравнения соболевского типа. - М.: Физматлит, 2007.

14. Соболев С. Л. Об одной новой задаче математической физики// Изв. АН СССР. Сер. мат. - 1954. 18. - C. 3-50.

15. Тихонов И. В., Әйделъман Ю. С. Вопросы корректности прямых и обратных задач для эволюционного уравнения специального вида// Мат. заметки. - 1994. - 56, № 2. - С. 99-113.

16. Тихонов И. В., Эйделъман Ю. С. Обратная задача для дифференциального уравнения в банаховом пространстве и распределение нулей целой функции типа Миттаг-Леффлера// Диффер. уравн. 2002. - 38, № 5. - С. 637-644.

17. Трибель $X$. Теория интерполяции. Функциональные пространства. Дифференциальные операторы. М.: Мир, 1980.

18. Уизем Д. Линейные и нелинейные волны. - М.: Мир, 1977.

19. Уразаева A. В., Федоров В. Е. Задачи прогноз-управления для некоторых систем уравнений гидродинамики// Диффер. уравн. - 2008. - 44, № 8. - С. 1111-1119.

20. Уразаева A. B., Федоров B. Е. О корректности задачи прогноз-управления для некоторых систем уравнений// Мат. заметки. - 2009. - 85, № 3. - С. 440-450.

21. Фалалеев M. В. Абстрактная задача прогноз-управление с вырождением в банаховых пространствах// Изв. Иркутск. гос. ун-та. Сер. мат. - 2010. - 3, № 1. - С. 126-132.

22. Федоров B. Е., Гордиевских Д. М. Разрешающие операторы вырожденных эволюционных уравнений с дробной производной по времени// Изв. вузов. Мат. - 2015. - № 1. - С. 71-83.

23. Федоров B. Е., Гордиевских Д. М., Плеханова М. В. Уравнения в банаховых пространствах с вырожденным оператором под знаком дробной производной// Диффер. уравн. - 2015. - 51, № 10. C. $1367-1375$.

24. Федоров B. E., Иванова Н. Д. Нелинейная эволюционная обратная задача для некоторых уравнений соболевского типа// Сиб. электрон. мат. изв. - 2011. - 8. - С. 363-378. 
25. Федоров B. E., Уразаева A. В. Линейная эволюционная обратная задача для уравнений соболевского типа// в кн.: Неклассические уравнения математической физики. - Новосибирск: Изд-во Ин-та математики им. С. Л. Соболева СО РАН, 2010. - С. 293-310.

26. Abasheeva N. L. Some inverse problems for parabolic equations with changing time direction// J. Inv. Ill-Posed Probl. — 2004. — 12, № 4. - P. 337-348.

27. Al Horani M., Favini A. Degenerate first-order inverse problems in Banach spaces// Nonlin. Anal. — 2012. - 175, № 1. - P. 68-77.

28. Caputo M. Lineal model of dissipation whose $Q$ is almost frequancy independent. II// Geophys. J. Astron. Soc. - 1967. - 13. - P. 529-539.

29. Favini A., Lorenzi A. Differential Equations. Inverse and Direct Problems. — New York: Chapman and Hall/CRC, 2006.

30. Favini A., Yagi A. Degenerate Differential Equations in Banach Spaces. — New York: Marcel Dekker, 1999.

31. Fedorov V. E., Ivanova N. D. Identification problem for a degenerate evolution equation with overdetermination on the solution semigroup kernel// Discr. Contin. Dynam. Syst. Ser. S. — 2016. — 9, № 3. — P. 687-696.

32. Fedorov V. E., Ivanova N. D. Identification problem for degenerate evolution equations of fractional order// Fract. Calc. Appl. Anal. — 2017. — 20, № 3. - P. 706-721.

33. Fedorov V. E., Urazaeva A. V. An inverse problem for linear Sobolev-type equations// J. Inv. Ill-Posed Probl. - 2004. - 12, № 4. - P. 387-395.

34. Kozhanov A. I. Composite Type Equations and Inverse Problems. - Utrecht: VSP, 1999.

35. Liu Y., Rundell W., Yamamoto M. Strong maximum principle for fractional diffusion equations and an application to an inverse source problem// Fract. Calc. Appl. Anal. — 2016. — 19, № 4. — P. 888-906.

36. Orlovsky D. G. Parameter determination in a differential equation of fractional order with RiemannLiouville fractional derivative in a Hilbert space// J. Sib. Federal Univ. Math. Phys. — 2015. — 8, № 1. — P. 55-63.

37. Plekhanova M. V. Nonlinear equations with degenerate operator at fractional Caputo derivative// Math. Meth. Appl. Sci. - 2016. - 40. - P. 41-44.

38. Prilepko A. I., Orlovskii D. G., Vasin I. A. Methods for Solving Inverse Problems in Mathematical Physics. - New York-Basel: Marcel Dekker, 2000.

39. Sviridyuk G. A., Fedorov V. E. Linear Sobolev Type Equations and Degenerate Semigroups of Operators. - Utrecht: VSP, 2003.

Федоров Владимир Евгеньевич

Челябинский государственный университет;

Шадринский государственный педагогический университет;

Южно-Уральский государственный университет

(национальный исследовательский университет), Челябинск

E-mail: kar@csu.ru

Нагуманова Анна Викторовна

Челябинский государственный университет

E-mail: urazaeva_anna@mail.ru 